\title{
Continuous frictional angular extrusion and its application in the production of ultrafine-grained sheet metals
}

\author{
Y. Huang ${ }^{\mathrm{a}, \mathrm{b}, *}$ and P.B. Prangnell ${ }^{\mathrm{b}}$ \\ ${ }^{a}$ Confae Technology Ltd., 30 Frieston Road, Timperley, Altrincham, Cheshire WA14 5AP, UK \\ ${ }^{\mathrm{b}}$ Manchester Materials Science Centre, The University of Manchester, Grosvenor Street, Manchester M17HS, UK
}

Received 26 October 2006; accepted 22 November 2006

Available online 18 December 2006

\begin{abstract}
A novel continuous severe deformation processing technique, continuous frictional angular extrusion (CFAE), has been developed for producing ultrafine-grained materials with high productivity. An investigation into the feasibility of this technique for processing sheet metals has been carried out using commercially pure aluminium. A uniform ultrafine-grained structure with an average grain size of $\sim 0.6 \mu \mathrm{m}$ was achieved in the material after a true strain of 6.6. The evolution and homogeneity of the deformation structure and texture during processing are reported.
\end{abstract}

(C) 2006 Acta Materialia Inc. Published by Elsevier Ltd. All rights reserved.

Keywords: Continuous frictional angular extrusion; SPD; Aluminium; Ultrafine-grained structure; EBSD

Severe plastic deformation (SPD) is an effective method for producing ultrafine-grained (UFG) metals with enhanced mechanical and physiochemical properties $[1,2]$. In the last decade or so, extensive research has been carried out in order to develop and optimize SPD techniques for commercial utilization. So far, of all the SPD techniques studied, equal channel angular extrusion (ECAE) [3] has attracted the most attention, due to its capability of producing UFG structures at scales large enough for structural applications [4,5]. However, the ECAE technique in its original form has limitations for commercial application. These include: (i) limited scalability; (ii) a high scrap rate; and (iii) large load requirements. These limitations, plus its batch nature, makes the ECAE process a technique that performs with low production efficiency.

Continuous processing is the key for an SPD technique that can produce large quantities of UFG metals with high productivity. Several attempts have been made to transform ECAE into a continuous process. For example, continuous constrained strip shearing (C2S2) [6] and ECAP-Conform [7] are recently reported methods for continuously processing sheets and rods to produce UFG structures. A common feature of these

\footnotetext{
* Corresponding author. Address: Confae Technology Ltd., 30 Frieston Road, Timperley, Altrincham, Cheshire WA14 5AP, UK; e-mail addresses: yan.huang-2@manchester.ac.uk; yan.huang@confae.com
}

processes is the adoption of the Conform technique [8] for feeding the work-piece by friction into a modified ECAE die assembly. Although these methods exhibit some useful features, they all use a long confined feeding passage for building up pressure on the work-piece and to generate sufficient frictional force to push the material through the die assembly. This causes several problems. For example, the work-piece undergoes a reduction in thickness, or shape change, before entry into the die's shear zone and consequently experiences a complicated deformation history, including redundant strains, and there is a resultant excessive requirement for torque. Furthermore, these techniques have difficulties with lubrication. Together, these problems impose practical limitations for scale-up.

Continuous frictional angular extrusion (CFAE) was developed with the aim of overcoming many of these problems so that large volumes of UFG alloys could be produced with high productivity (pending UK patent [9]). The CFAE technique (illustrated in Fig. 1) involves the use of an ECAE-based die assembly combined with several innovative steps, including the use of a frictional driving roll, which applies a normal pressure to the work-piece without any plastic compression, and reduced friction within the die. Thus, continuous processing is possible with plastic deformation occurring principally by shear without changing the dimensions of the work-piece. Ultra-high strains can be achieved 


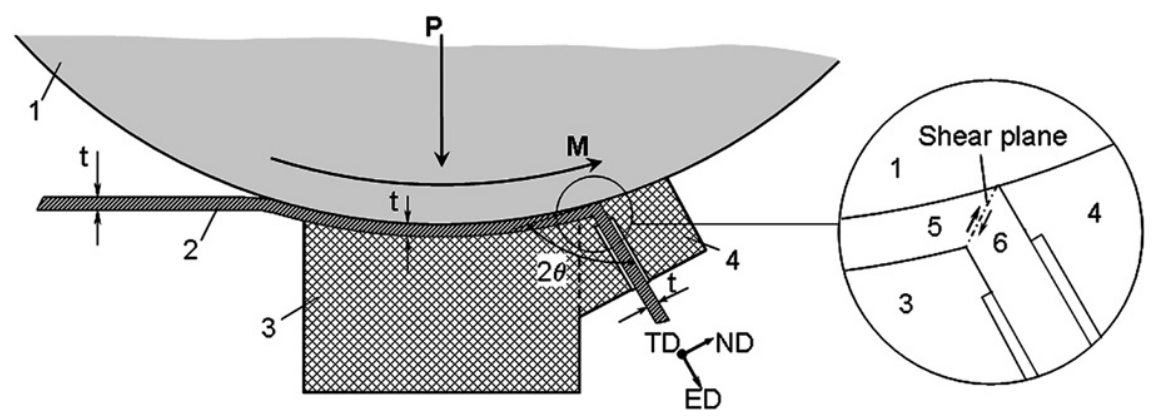

Figure 1. Schematic diagram of the CFAE set-up: 1 , driving roll; 2 , sheet work-piece, 3 , work-piece support block; 4 , die assembly, where $2 \theta$ is the extrusion angle; 5 , first extrusion channel; 6 , second extrusion channel. The reference system used to describe the microstructures and textures is also shown: ED, extrusion direction; ND, normal to the sheet; and TD, transverse direction.

through repetition of the process. Theoretically the CFAE technique offers several benefits over existing methods, including: (i) being suitable for processing large quantities of material (including sheet, bar and rod); (ii) a high productivity and a low scrap rate; and (iii) less extrusion force - due to minimized die friction and the elimination of the work-piece plastic compression in the first channel. The purpose of the present work was to study experimentally the feasibility of the CFAE technique for processing UFG structured metals in a sheet form, using a modified rolling mill.

A two-high rolling mill was adapted for CFAE processing of sheet samples, as illustrated in Figure 1. The top roll functioned as the driving roll (1), rotating to provide a torque $(M)$ and at the same time applying a pressing force $(P)$ to the work-piece (2). A work-piece support block (3) with an attached die assembly (4) was introduced by replacing the bottom roll. The working surface of the work-piece support block was matched with the curvature of the sheet on the driving roll and formed the first extrusion channel (5). The second extrusion channel (6) was a short slot, to minimize friction, in the stationary die assembly. The second extrusion channel was inclined at an extrusion angle of $2 \theta$. Both extrusion channels had an identical cross-section, which matched the sheet thickness $(t)$. Similar to ECAE, under ideal simple shear conditions the effective plastic strain $\left(\varepsilon_{\mathrm{vm}}\right)$ that the work-piece undergoes through the die assembly is dependent only on the extrusion angle $(2 \theta)$, given by $\varepsilon_{\mathrm{vm}}=1.15 \cot \theta$ [3]. If the friction in the second extrusion channel is negligible, the extrusion pressure $(p)$ is a function of the material strength $\left(\sigma_{\mathrm{s}}\right)$ and the extrusion angle as $p=1.15 \sigma_{\mathrm{s}} \cot \theta[3]$.

The sheet samples $(2 \times 30 \times 2000 \mathrm{~mm})$ of cold rolled (H Temper) commercial purity aluminum AA1050 (cut in the rolling direction) were processed by CFAE. Processing was carried out at room temperature at $0.09 \mathrm{~m} \mathrm{~s}^{-1}$. The sheets were processed to up to 10 passes (equivalent true strain of 6.6), with the sheet orientation maintained constant throughout. In order to ensure that the frictional coefficient between the driving roll and the work-piece was significantly larger than that between the work-piece and the support block, which is an essential requirement for the functioning of the process, a diamond-like carbon coating was applied to the surfaces of the support block and the second extrusion channel, and the driving roll was chemically and mechanically roughened. A combination of PTFE spray and colloidal graphite was used for lubricating the bottom surface of the work-piece. The top surface of the work-piece, which was in contact with the driving roll, underwent cleaning to ensure sufficient frictional force for extrusion.

The deformation structures of the CFAE-processed sheets were examined in a field emission gun scanning electron microscope (FEGSEM) and using high-resolution electron backscattered diffraction (EBSD) on the longitudinal ND-ED/RD plane (see Fig. 1). EBSD orientation maps were acquired at different strain levels with spatial and angular resolution of $\sim 50 \mathrm{~nm}$ and $\sim 1^{\circ}$. EBSD data was analysed by $\mathrm{VMAP}^{\odot}$ software. In the data presented, high-angle boundaries (HABs) and low-angle boundaries (LABs) are defined as having misorientations greater than or equal to $15^{\circ}$ and less than $15^{\circ}$, respectively. Due to misorientation noise, boundaries of less than $1.5^{\circ}$ misorientation were cut off. Crystallographic textures were determined by EBSD from a minimum of 10,000 orientations over an area of $500 \times 500 \mu \mathrm{m}$.

Using the set-up illustrated in Figure 1, CFAE processing performed successfully under a range of conditions. Figure 2 shows examples of the deformation
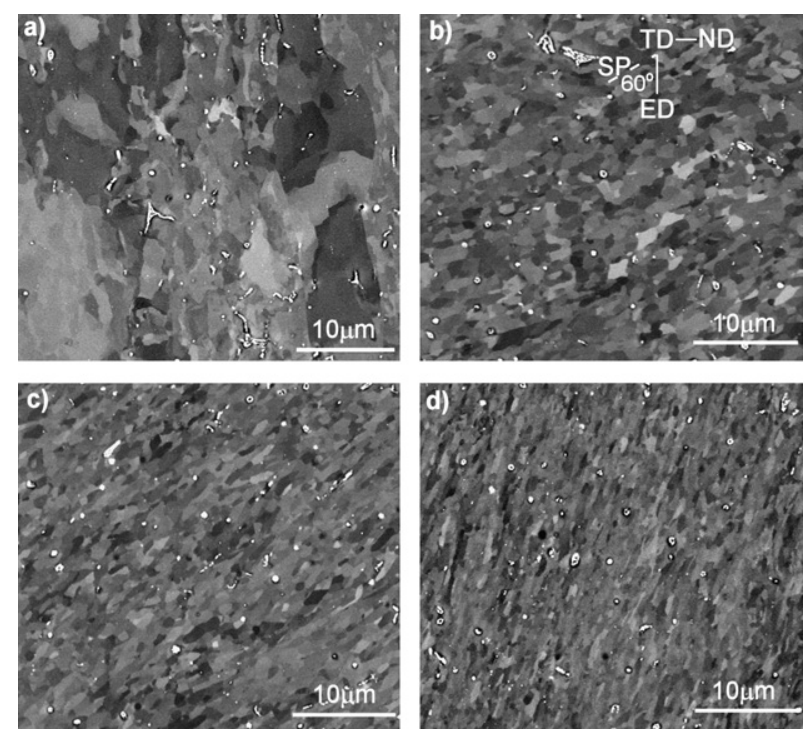

Figure 2. FEGSEM backscattered images showing the deformation structures of AA1050 sheet samples processed to various strains: (a) the initial cold rolled structure; (b) $\varepsilon_{\mathrm{vm}} \sim 0.66$ (one pass); (c) $\varepsilon_{\mathrm{vm}} \sim 2.7$ (four passes); (d) $\varepsilon_{\mathrm{vm}} \sim 6.6$ (10 passes). 
structures obtained after CFAE processing to different levels of strain. The starting material was cold rolled with a coarse subgrain structure (Fig. 2a). After 1 CFAE pass $\left(\varepsilon_{\mathrm{vm}} \sim 0.66\right)$, a uniform and finer subgrain structure formed from the initial coarse cold-rolled structure with cell bands aligned along the shear plane, which was $60^{\circ}$ to the vertical extrusion direction (Fig. 2b). With further passes, a fibrous deformation structure started to form along a direction between the die shear plane and the extrusion direction. As the strain increased, the fibrous structure compressed in transverse spacing, became more uniform and rotated towards the extrusion direction (Fig. 2c and d).

Deformation was found to be reasonably uniform throughout the thickness of the work-piece and the effects of surface friction were limited (Fig. 3). A significant surface friction affected zone, with a depth of $\sim 100 \mu \mathrm{m}$, was only seen where the sheet contacted the stationary bottom die surface (Fig. 3a). At the sheet's top roll contacted surface (Fig. 3c) the microstructure was almost indistinguishable from the matrix (Fig. 3b). This phenomenon is a characteristic feature of the CFAE process, due to fact that the sheet's bottom surface experiences sliding contact with the support block while the top surface moves at the same speed as the driving roll.

Example EBSD maps are shown in Figure 4, illustrating the development of HABs with strain. The starting cold rolled material had a mean $\mathrm{HAB}$ spacing of $\sim 35 \mu \mathrm{m}$ in the coarse subgrain matrix (Fig. 4a). After four CFAE passes $\left(\varepsilon_{\mathrm{vm}} \sim 2.65\right)$, a lamellar boundary structure developed and the average spacing of HABs, or grain width, was about $1.5 \mu \mathrm{m}$ (Fig. 4b). After eight CFAE passes $\left(\varepsilon_{\mathrm{vm}} \sim 5.3\right)$, the average grain width reached a submicron scale and in most regions groups of elongated submicron grains were seen to have formed within the lamellar structure (Fig. 4c). Following 10 CFAE passes $\left(\varepsilon_{\mathrm{vm}} \sim 6.6\right)$, the deformation structure can be seen to have developed into a reasonably uniform submicron, or UFG structure (Fig. 4d), with an average grain size of about $0.6 \mu \mathrm{m}$ and an average aspect ratio (grain length/width) of 1.7, although it still contained some unrefined bands. Detailed EBSD measurements
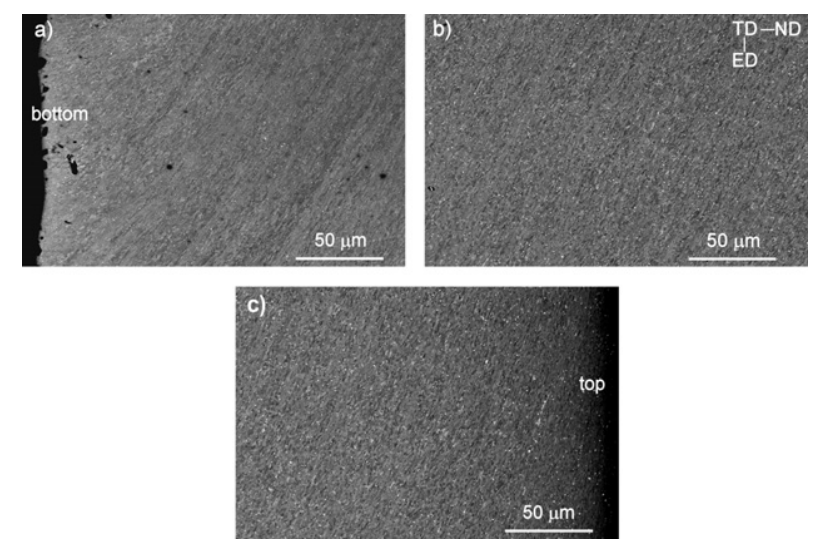

Figure 3. FEGSEM backscattered images showing the uniformity of the microstructure through the thickness of a sample processed to eight passes: (a) bottom surface; (b) centre and (c) top surface.
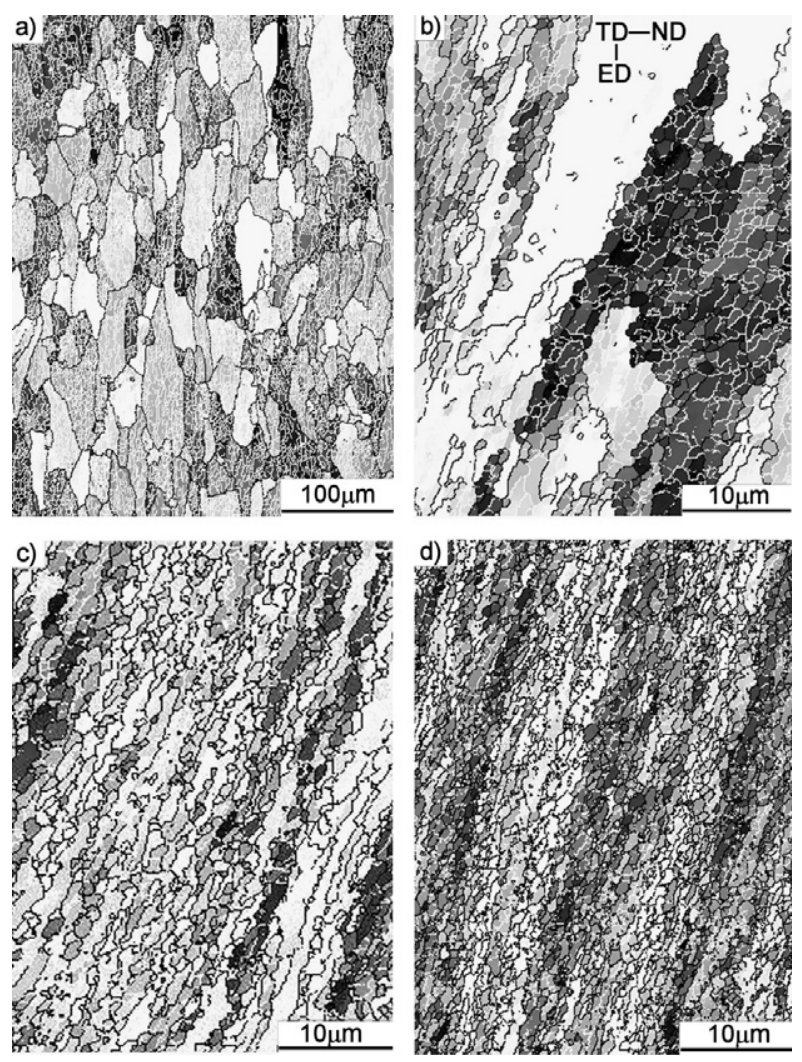

Figure 4. EBSD maps showing the development of grain boundary structures with strain: (a) $\varepsilon_{\mathrm{vm}}=0$; (b) $\varepsilon_{\mathrm{vm}} \sim 2.7$ (four passes); (c) $\varepsilon_{\mathrm{vm}} \sim 5.3$ (eight passes); (d) $\varepsilon_{\mathrm{vm}} \sim 6.6$ (10 passes). Black lines represent high-angle grain boundaries $\left(>15^{\circ}\right)$ and white lines low-angle boundaries $\left(1.5-15^{\circ}\right)$.

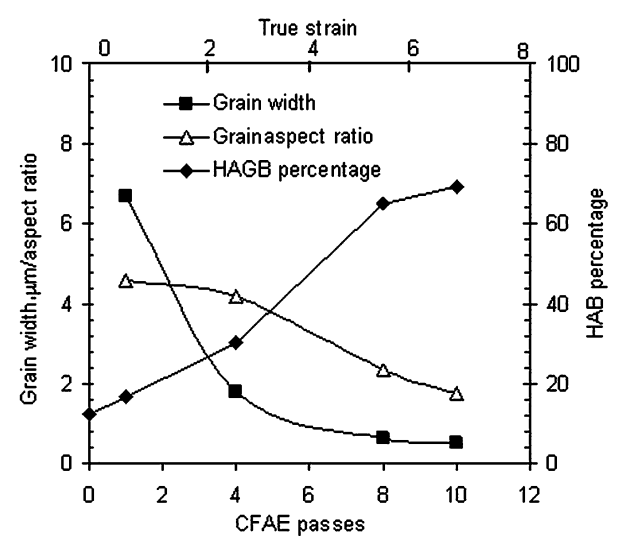

Figure 5. Statistical data from EBSD measurements, showing grain width, aspect ratio and the percentage of $\mathrm{HAB}$ area as a function of CFAE pass of up to $10\left(\varepsilon_{\mathrm{vm}} \sim 6.6\right)$.

of grain width, aspect ratio and fraction of HAGs are given in Figure 5 as a function of CFAE pass (strain). The high-resolution EBSD statistical data (Fig. 5) revealed that a significant fraction of HABs developed after four CFAE passes and HABs became dominant after eight CFAE passes, with a fraction of $65 \%$; an $\mathrm{HAB}$ fraction of $\sim 70 \%$ was obtained after $10 \mathrm{CFAE}$ passes. The final $\mathrm{HAB}$ area of $70 \%$ is sufficient to form a stable HAB network without discontinuous 

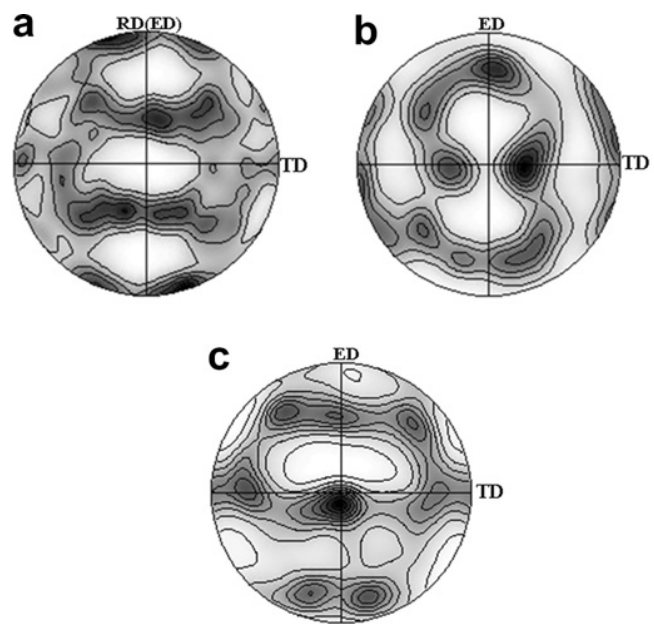

Figure 6. 111 pole figures, showing the texture evolution with CFAE passes: (a) the initial rolling texture; (b) after one pass and (c) after eight passes.

recrystallization on annealing [10]. The overall trends in the statistical data are in agreement with previous ECAE studies [11].

Figure 6 shows 111 pole figures of the starting material and the deformation textures after one and eight CFAE passes. After one CFAE pass, the initial rolling texture (Fig. 6a), which included $S\{123\}\langle 634\rangle$, brass $\{011\}\langle 112\rangle$, cube $\{001\}\langle 100\rangle$ and $P\{011\}\langle 122\rangle$ components, weakened (shown in Fig. 6b) and, with reference to the sheet plane\} $\langle\mathrm{ED}\rangle$, a simple shear texture $\{111\}\langle 110\rangle$ and rotated cube texture $\{100\}\langle 110\rangle$ began to form. This evolution continued with increasing strain and after four CFAE passes the initial texture disappeared. After eight CFAE passes (Fig. 6c), textures associated purely with CFAE deformation developed. The main texture components were, approximately, shear texture $\{111\}\langle 110\rangle$ and rotated cube $\{100\}$ $\langle 110\rangle$, and the textures were broadly similar those found in face-centred cubic metals processed by ECAE [12] and $\mathrm{C} 2 \mathrm{~S} 2$ [6].

It is well known that the formability of metal sheets is texture dependent [13]. Textures with $\{111\}$ planes parallel to the sheet plane enhance formability, whereas textures with $\{001\}$ planes parallel to the sheet plane have the opposite effect $[13,14]$. In this context, the development of the observed $\{111\}\langle 110\rangle$ shear texture and the weakening of the cube texture $\{001\}\langle 100\rangle$ dur- ing CFAE processing is advantageous because it will help to improve formability of the material.

In summary, this study has shown that CFAE is an effective method for producing UFG metal sheets with potentially high productivity, although more investigation is needed to verify its advantageous features with larger-scale processing. CFAE processing of AA1050 sheet samples was successfully carried out to various total numbers of passes and a uniform and nearly equiaxed UFG structure was achieved after an equivalent strain of 6.6. The evolution of deformation structure and texture during CFAE processing was examined using the EBSD technique and was found to be similar to that seen in ECAE. The textures developed were consistent with those expected to improve formability in metal sheets.

The authors acknowledge the financial support of the Department of Trade and Industry, UK (GONW8080TEC) and the University of Manchester EPSRC Light Alloys Portfolio Partnership (EP/ D029201/1) for this project.

[1] R.Z. Valiev, N.A. Krasilnikov, N.K. Tsenev, Mater. Sci. Eng. A 137 (1991) 35.

[2] F.J. Humphreys, P.B. Prangnell, J.R. Bowen, A. Gholinia, C. Harris, Phil. Trans. R. Soc. Lond. A 357 (1999) 1663.

[3] V.M. Segal, Mater. Sci. Eng. A 197 (1995) 157.

[4] R.Z. Valiev, R.K. Islamgaliev, I.V. Alexandrov, Prog. Mater. Sci. 45 (2000) 103.

[5] V.M. Segal, Mater. Sci. Eng. A 386 (2004) 269.

[6] J-C. Lee, H-K. Seok, J-H Han, Y-H. Chung, Mater. Res. Bull. 36 (2001) 997.

[7] G.J. Raab, R.Z. Valiev, T.C. Lowe, Y.T. Zhu, Mater. Sci. Eng. A 382 (2004) 30.

[8] D. Green, Atomic Energy Authority UK, UK patent GB1370894, 1973.

[9] Y. Huang, UK patent GB2490997A, 2005.

[10] P.B. Prangnell, J.S. Hayes, J.R. Bowen, P.J. Apps, P.S. Bate, Acta Mater. 52 (2004) 3193.

[11] P.B. Prangnell, J.R. Bowen, P.J. Apps, Mater. Sci. Eng. A 373-377 (2004) 178.

[12] W. Huang, L. Chang, P.W. Hao, C.P. Chang, Mater. Sci. Eng. A 307 (2001) 87.

[13] M. Hatherly, W.B. Hutchinson, An Introduction to Textures in Metals, Institution of Metallurgists, London, 1979.

[14] J. Hirsch, K. Lucke, Acta Metall. 36 (1988) 2883. 